\title{
BMJ Open Observational stepped-wedge analysis of a community health worker-led intervention for diabetes and hypertension in rural Mexico
}

\author{
Devin T Worster (D) , , ${ }^{1,2}$ Molly F Franke (D) , ${ }^{3}$ Rodrigo Bazúa, ${ }^{2}$ Hugo Flores, ${ }^{1,2}$ \\ Zulema García, ${ }^{2}$ Joanna Krupp, ${ }^{2}$ Jimena Maza, ${ }^{2}$ Lindsay Palazuelos, ${ }^{4}$ \\ Katia Rodríguez, ${ }^{2}$ Patrick M Newman, ${ }^{2,5}$ Daniel Palazuelos ${ }^{1,2,3,4}$
}

To cite: Worster DT, Franke MF, Bazúa R, et al. Observational stepped-wedge analysis of a community health worker-led intervention for diabetes and hypertension in rural Mexico. BMJ Open 2020;10:e034749. doi:10.1136/ bmjopen-2019-034749

- Prepublication history and additional material for this paper are available online. To view these files, please visit the journal online (http://dx.doi. org/10.1136/bmjopen-2019034749).

PMN and DP are joint senior authors.

Received 04 October 2019 Revised 14 January 2020 Accepted 27 January 2020

Check for updates

(C) Author(s) (or their employer(s)) 2020. Re-use permitted under CC BY-NC. No commercial re-use. See rights and permissions. Published by BMJ.

For numbered affiliations see end of article.

Correspondence to

Dr Devin T Worster;

dtw4001@med.cornell.edu

\section{ABSTRACT}

Objectives There is emerging interest and data supporting the effectiveness of community health workers (CHWs) in non-communicable diseases (NCDs) in low/ middle-income countries (LMICs). This study aimed to determine whether a CHW-led intervention targeting diabetes and hypertension could improve markers of clinical disease control in rural Mexico.

Design and setting A prospective observational steppedwedge study was conducted across seven communities in rural Chiapas, Mexico from March 2014 to April 2018.

Participants 149 adults with hypertension and/or diabetes.

Intervention This study was conducted in the context of the programmatic roll-out of an accompanimentbased $\mathrm{CHW}$-led intervention designed to complement comprehensive primary care for adults with diabetes and/ or hypertension. Implementation occurred sequentially at 3-month intervals with point-of-care data collected at baseline and every 3 months thereafter for 12 months following roll-out in all communities.

Outcome measures Primary outcomes were glycated haemoglobin (HbA1c) and systolic blood pressure (SBP), overall and stratified by baseline disease control. We conducted an individual-level analysis using mixed effects regression, adjusting for time, cohort and clustering at the individual and community levels.

Results Among patients with diabetes, the CHW-led intervention was associated with a decrease in $\mathrm{HbA1c}$ of $0.35 \%$; however, Cls were wide $(95 \% \mathrm{Cl}-0.90 \%$ to $0.20 \%$ ). In patients with hypertension, there was a $4.7 \mathrm{~mm} \mathrm{Hg}$ decrease in SBP $(95 \% \mathrm{Cl}-8.9$ to -0.6$)$. In diabetic patients with $\mathrm{HbA} 1 \mathrm{c} \geq 9 \%, \mathrm{HbA} 1 \mathrm{c}$ decreased by $0.96 \%(95 \% \mathrm{Cl}-1.69 \%$ to $-0.23 \%)$, and in patients with uncontrolled hypertension, SBP decreased by $10.2 \mathrm{~mm} \mathrm{Hg}$ $(95 \% \mathrm{Cl}-17.7$ to -2.8$)$.

Conclusions We found that a CHW-led intervention resulted in clinically meaningful improvement in disease markers for patients with diabetes and hypertension, most apparent among patients with hypertension and patients with uncontrolled disease at baseline. These findings suggest that $\mathrm{CHWs}$ can play a valuable role in supporting NCD management in LMICs.

Trial registration number NCT02549495.
Strengths and limitations of this study

- This study evaluates a community health worker (CHW)-led intervention, versus comprehensive primary care alone, on measures of clinical disease control among patients with non-communicable diseases in Latin America.

- We expand on a prior analysis to quantify the effect of the CHW intervention on clinical indicators of diabetes and hypertension control in an expanded cohort of communities and patients, examining whether the intervention performed differently in patients with poor disease control.

- Using the programmatic stepped roll-out of the CHW intervention allowed for individual-level analysis as in a stepped-wedge trial, limiting confounding by stable individual-level characteristics.

- The stepped-wedge design is a practical approach for implementation and evaluation of low-risk interventions expected to confer a large benefit in impoverished settings, limiting the ethical issue of non-treatment common to randomised trials.

- Limitations of this study include small sample size, which may have impacted the precision of several analyses, and evaluation in a single rural, remote region. However, the successes of the intervention in spite of numerous barriers and mounting comparable evidence from low/middle-income countries suggest that the findings may be generalisable to other remote, rural settings.

\section{INTRODUCTION}

Seventy-one per cent (41 million) of all deaths worldwide are attributable to noncommunicable diseases (NCDs) including cardiovascular disease and diabetes. Over three-quarters (32 million) of these occur in low/middle-income countries (LMICs). ${ }^{1}$ Poor patients suffer a higher burden of NCD risk factors and worse outcomes, with over $85 \%$ of premature deaths from NCDs occurring in LMICs. ${ }^{12}$ 
Community health workers (CHWs) have the potential to play a significant role in strengthening health systems worldwide, with increasing interest in their support for NCD management and emerging evidence of their effectiveness. ${ }^{3}{ }^{4}$ Understanding the effect of CHW interventions on biologic markers of disease control is important to help anticipate the effects of successful programmes on individual and population health. A $1 \%$ reduction in glycated haemoglobin (HbAlc) among patients with diabetes or a $10 \mathrm{~mm} \mathrm{Hg}$ reduction in systolic blood pressure (SBP) in patients with hypertension has been associated with a reduction in disease-related deaths as well as microvascular and macrovascular complications. ${ }^{56}$

Until recently, evidence for the ability of CHWs to improve NCD control in LMICs was limited. A recent systematic review of CHWs in LMICs for prevention and management of diabetes found positive outcomes in a majority of studies, but identified significant heterogeneity among structures of CHW interventions and limitations due to study design, high rates of attrition, absence of detailed reporting on operational design and variable programme fidelity. ${ }^{7}$ Observational studies of CHWled interventions demonstrated improvement in fasting plasma glucose,${ }^{89}$ though a recent cluster-randomised trial found inconclusive results, potentially due to a lack of power. ${ }^{10}$ For cardiovascular disease, two systematic reviews, including one meta-analysis of randomised trials, identified improvements in blood pressure with CHW interventions. ${ }^{411}$ Several recent cluster-randomised trials around the world demonstrated blood pressure reduction and improved cardiovascular risk control among patients with uncontrolled hypertension. ${ }^{1012-15}$ Variability in findings across studies could result from heterogeneity in CHW interventions, including CHW roles, which may include disease screening, individual or group disease education, lifestyle management, medication and clinic adherence support and medication management-with or without assistance of clinic physicians.

In Mexico, ischaemic heart disease and diabetes are the two leading causes of mortality. ${ }^{16}{ }^{17}$ A 2016 national survey estimated that $9.4 \%$ and $26 \%$ of Mexican adults had diabetes and hypertension, respectively, ${ }^{18}$ with low rates of clinical control and increasing prevalence across most demographics. ${ }^{17-19}$ The Mexican Ministry of Health $(\mathrm{MOH})$ identifies NCDs and corresponding risk factors as a priority and recognises the need for scalable, evidencebased interventions that address promotion of healthy behaviours and disease management. ${ }^{19}$ While there is no national-level CHW programme in Mexico, national strategies emphasise active community participation in addressing the rising burden of NCDs, advocate for the creation of community committees and encourage partnership with non-governmental organisations. ${ }^{17} 19$ Experience using Mexican Promotores de Salud as CHWs focussed on education and lifestyle modification has been varied but promising, ${ }^{20}$ though they have not yet been widely mobilised to provide individual or instrumental support in NCD management. Compañeros en Salud (CES) is an affiliate of the multinational non-governmental organisation Partners in Health. CES works in collaboration with the Mexican MOH in Chiapas, Mexico, a state with the highest rates of poverty, including extreme poverty, and one of the lowest rates of effective health coverage in the country. ${ }^{21}$ We previously found that a CHW intervention led to improved medication adherence and disease control among patients with diabetes and hypertension. ${ }^{22}$ Here we expand on this prior analysis to quantify the effect of the intervention on clinical indicators of diabetes and hypertension control in an expanded cohort of communities and patients, and examine whether the intervention performed differently in patients with poor disease control. We hypothesised that the intervention would reduce HbA1c in patients with diabetes and SBP in patients with hypertension.

\section{METHODS \\ Study design}

This study was structured around the planned programmatic roll-out of a CHW intervention targeting diabetes and hypertension in seven rural, remote communities (population 1000-2500) in Chiapas, Mexico where CES operates. To ensure sufficient time for implementation, including training and supervision of CHWs, the intervention was sequentially implemented at 3-month intervals. The first cohort included four communities in which CES had been working for 2 years. Study enrollment took place during March 2014 with intervention implementation at 3-month intervals and data collection through January 2016 (figure 1), and we found improved disease control and medication adherence among all patients. ${ }^{22}$ However, due to the small sample, the analysis combined patients with diabetes and hypertension and we were unable to quantify the disease-specific clinical effects of the intervention. Based on these findings, the intervention was subsequently scaled to three additional communities in which CES had been supporting primary care for 2years. Enrollment took place during July 2016, with implementation and data collection in this cohort through April 2018. In both cohorts, data collection took place at baseline (ie, prior to implementation in any community) and every 3 months thereafter for 12 months following implementation of the intervention in all seven communities (figure 1).

Participants provided verbal informed consent, which was documented in writing by study staff.

\section{Intervention}

CES has partnered with the Mexican MOH since February 2012 to provide comprehensive primary care and management for patients with NCDs, in accordance with national guidelines. The care model includes monthly clinic visits, disease counselling and treatment with common oral medications for diabetes and hypertension, which are provided free-of-charge to patients. 


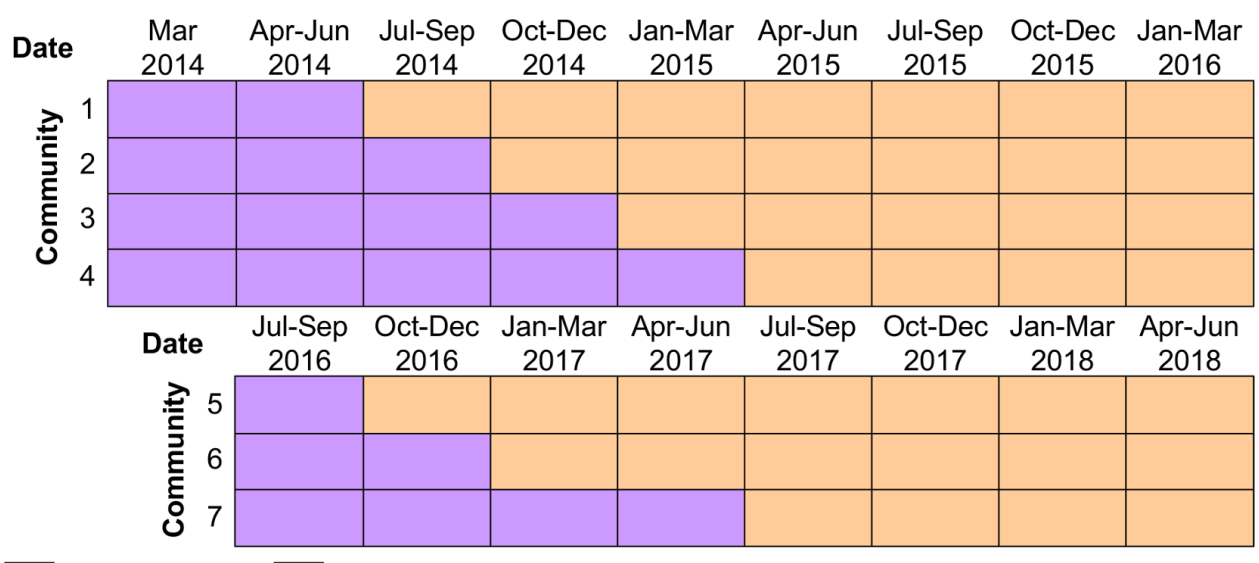

Pre-intervention $\square$ Intervention

Figure 1 Stepped-wedge schematic for the study. Programmatic roll-out was randomised by community (cluster) with sequential implementation of the intervention resulting in each community contributing time as unexposed (purple) and exposed (peach) to the CHW-led intervention. Data were collected at the start of each 3-month time point across two cohorts regardless of whether the intervention had been implemented. In the first cohort (communities 1-4), data collection took place from March 2014 through January 2016. In the second cohort (communities 5-7), data collection took place from July 2016 through April 2018. Delays in baseline data collection in cohort 1 shortened the duration of period 1 from 3 months to 1 month. Organisational delay in roll-out for cohort 2 shortened the baseline pre-randomisation phase and resulted in a 3-month delay in implementation in community 7 .

The CHW-led intervention follows a community-based accompaniment approach centred on regular home visits. This approach has demonstrated effectiveness in improving disease outcomes and medication adherence in patients with HIV. ${ }^{23}{ }^{24}$ CHWs serve as a bridge between patient and clinic, promoting medication adherence, reinforcing basic disease education, providing psychosocial support and supporting active case retention. The CHWs in this intervention are women who were nominated at community meetings (by self or community) and selected based on a formal interview process focussing on leadership potential, motivation, basic literacy and education. They were trained in four-times-weekly group sessions for 1 month, covering basic pathophysiology, diagnosis and treatment of chronic diseases including diabetes and hypertension, as well as practical training on the elements of a home visit and the logistical requirements of the role. They also participated in monthly refresher training sessions, covering themes such as motivational interviewing, recognition of emergencies and complications and navigation of interactions with challenging patients. CHWs work longitudinally with patients, conducting home visits which begin weekly then change in frequency based on a collaborative assessment of the patient's needs by the CHW and clinic physician with a minimum of one visit monthly. Home visits consist of disease counselling with motivational interviewing, assessment of medication adherence and supply and disease monitoring including blood pressure and capillary glucose measurement. CHWs accompany patients to clinic visits and meet regularly with clinic physicians to discuss patient management. They are compensated with household food and consumable items, worth a dollar amount approximately equivalent to a national conditional cash transfer programme in place at the time of the programme's creation. The overall structure of the CHW-led intervention was the same in both cohorts.

\section{Study participants}

Patients with diabetes, hypertension and respective risk factors were identified via a CES programme of clinicbased and door-to-door case finding. Each community has one health centre staffed by a social service general physician who maintains registries of patients with NCDs, which served as the basis for eligibility determination and recruitment. Eligible patients were those who had a diagnosis of diabetes and/or hypertension, were aged 18 years or older at the time of enrollment, resided in a study community and were prescribed daily medications by the clinic physician for treatment of diabetes and/ or hypertension. We excluded patients with secondary hypertension, type 1 diabetes, pregnancy and chronic use of glucocorticoids. We also excluded patients who, after enrollment but prior to implementation of the intervention in the first community, were removed from treatment by their physician, moved outside the study community, transferred care to another health facility or who were determined not to have a diagnosis of diabetes or hypertension.

\section{Patient and public involvement}

Patients and the public were not involved in the design, conduct or reporting of this study.

\section{Data collection}

Study staff visited patient homes and collected data at baseline and 3-month intervals thereafter, timed just prior to roll-out of the intervention in a new community. At enrollment, we administered a basic demographic and socioeconomic questionnaire to all patients. Outcomes 
data measured included SBP and diastolic blood pressure (DBP) (in $\mathrm{mm} \mathrm{Hg}$ ) among patients with hypertension and HbAlc (in \% HbAlc) among patients with diabetes. We measured blood pressure in a seated position using two measurements (bilateral arms) with the Omron HEM 7080IT E automated blood pressure cuff, taking a third measurement if the difference in SBP was $\geq 6 \mathrm{~mm}$ $\mathrm{Hg}$. Average SBP and DBP were calculated as the mean between the two closest readings. We measured HbAlc using the Bayer Alc NOW point-of-care device. Adverse effects and clinical events (death, myocardial infarction, stroke) were recorded at 3-month intervals.

\section{Outcomes}

The primary outcomes for this study were HbAlc and SBP, analysed as continuous variables. These were analysed among all patients with diabetes and hypertension, respectively, and in subgroups characterised by level of disease control at baseline to assess for effect modification by baseline level of control. We hypothesised that the intervention may offer greater clinical benefit in patients without consistent disease control. For example, while improvement in markers of disease control would be considered a successful outcome among patients with uncontrolled disease, maintenance of disease control, but not necessarily improvement, would be considered a successful outcome among patients with controlled disease. Though not pre-specified by protocol, this stratified analysis was agreed on by investigators based on clinical applicability prior to initiation of data analysis. Poor diabetes control at baseline was defined as HbAlc $\geq 9 \%$, reflecting the standard of various quality metrics. ${ }^{25}$ Disease control among patients with hypertension was defined according to Mexican national guidelines: blood pressure $<140 / 90 \mathrm{~mm} \mathrm{Hg}$ for patients with hypertension and no diabetes; blood pressure $<130 / 80 \mathrm{~mm} \mathrm{Hg}$ for patients with hypertension and diabetes; and blood pressure $<150 / 90 \mathrm{~mm} \mathrm{Hg}$ for patients over the age of $80 .{ }^{17}$ Secondary outcomes included DBP, analysed as a continuous variable, and disease control examined as a binary variable (ie, $\mathrm{HbA1c}<7 \%$ for diabetic patients per national guidelines and the above thresholds for hypertensive patients).

\section{Statistical methods and data analysis}

The study size was limited by the number of patients meeting eligibility criteria in the communities where the intervention was to be implemented. We therefore calculated detectable alternatives with at least $80 \%$ power using a sample size calculator that allowed for clustering at the individual and community levels. ${ }^{26} \mathrm{We}$ assumed an intraperson correlation of 0.7 and intracommunity correlation of 0.05 . For expected enrollment of 70 patients with diabetes, we would have $>80 \%$ power to detect a difference in HbAlc of $\pm 0.3 \%$, assuming a baseline HbA1c of 9.5 (SD 2.5). Assuming 110 patients with hypertension, we would have $>80 \%$ power to detect a difference in SBP of $\pm 2 \mathrm{~mm} \mathrm{Hg}$, assuming a baseline SBP of $140 \mathrm{~mm} \mathrm{Hg}$ (SD 20). Calculations assumed an alpha of 0.05 .

Outcomes assessments for individuals who withdrew from the study for any reason were included until the time at which they withdrew. We conducted individuallevel mixed effects analyses including random intercepts for each individual and community to adjust variances for individual repeated measures and clustering by community, respectively. ${ }^{27-29}$ The random intercept for community was excluded from the model if the variance for the intercept was zero to avoid overfitting. We modelled continuous outcomes (HbA1c, SBP, DBP) using linear mixed models with maximum likelihood estimation. ${ }^{27} 29$ Binary outcomes were modelled using generalised linear mixed models with Laplace maximum likelihood estimation and a logit link. ${ }^{28} 30$ Fixed effects included a binary variable to indicate whether the person lived in a community that was exposed to the intervention at a given time point, a categorical variable for time (ie, corresponding to each intervention/data collection step) to adjust for secular trends and an indicator variable for cohort (first vs second). We conducted stratified analyses to examine whether any effect of the intervention depended on baseline disease control and calculated $\mathrm{p}$ values for differences in continuous outcomes and ORs using Cochran's Q-test for heterogeneity. We calculated the intraperson (intracluster) correlation (ICC) of continuous outcomes for patients with two HbA1c or blood pressure measurements collected when unexposed to the intervention.

We performed multiple sensitivity analyses. To examine whether regression to mean could explain the findings of stratified analyses, we repeated stratified analyses removing the baseline value. Because regression to the mean is caused by random error, the measurement following the baseline measurement (ie, that used to define disease control) would be expected to be closer to the population mean. Thus, if findings of stratified analyses remain similar after excluding the baseline measurement, regression to the mean would be less of a concern. We additionally assessed primary and secondary outcomes using 2017 Mexican MOH guidelines for hypertension featuring more liberal blood pressure targets: $<140 / 80$ in patients with hypertension and diabetes, $<150 / 90$ if over the age of 60 with hypertension and without diabetes and $<140 / 90$ if over the age of 60 with hypertension and diabetes. ${ }^{31}$ To assess for varying trends across communities, we adjusted for community as a fixed effect. We also conducted an analysis in which we modelled time as a random effect with slopes for each cluster (community, individual). ${ }^{29}$ Finally, we excluded 11 patients who were removed from treatment by their providers during the study.

Analyses were conducted using SAS V.9.4 (Cary Institute). 


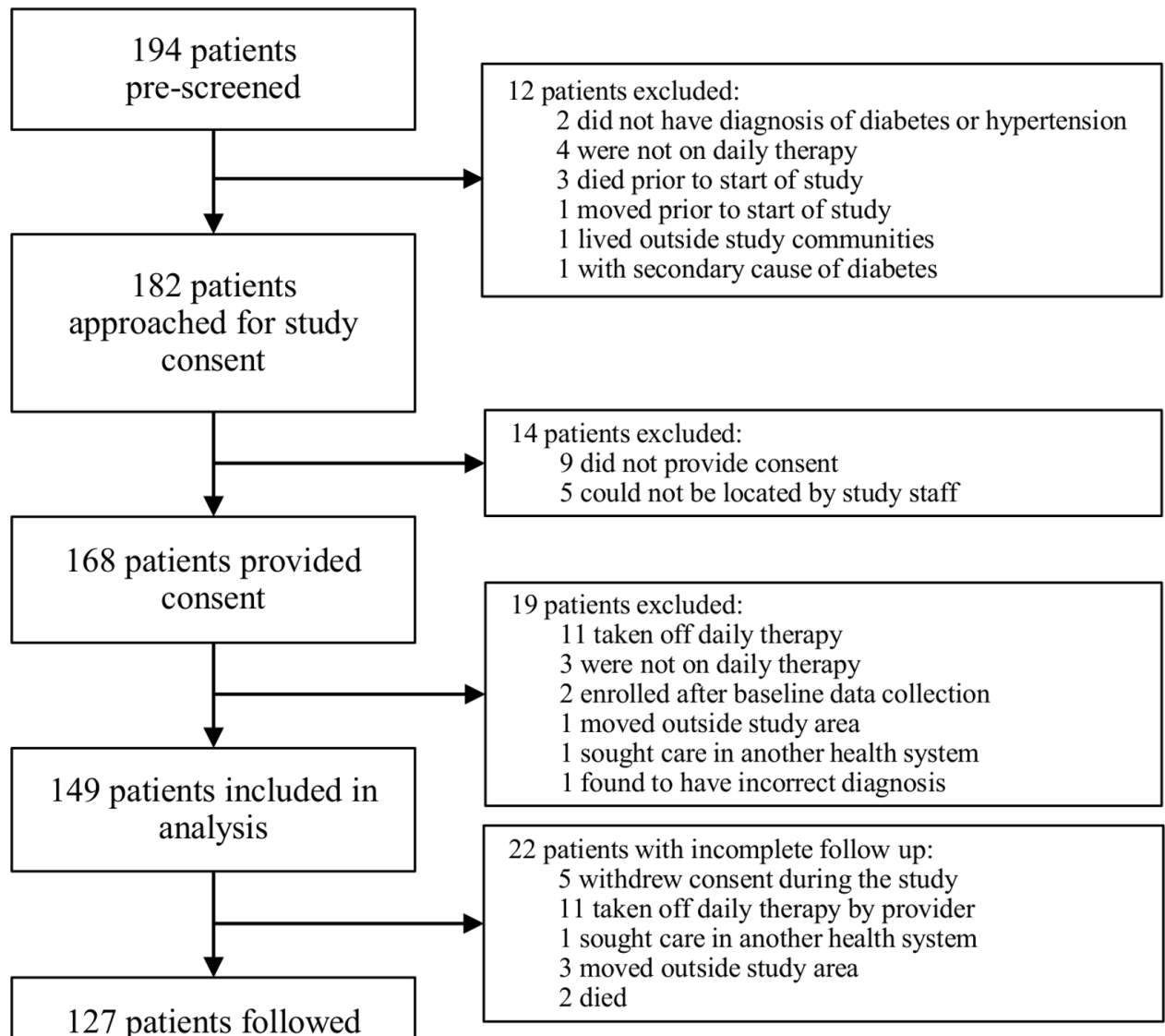

through end of study

Figure 2 Flow of participants through the study.

\section{RESULTS}

\section{Description of the study cohort and data completeness}

We screened 194 patients identified through clinic NCD registries and enrolled 168 patients who provided informed consent in March 2014 (cohort 1) and July 2016 (cohort 2; figure 2). Nineteen patients (11\%) were excluded prior to intervention or analysis. Of the 149 patients analysed, 39 (26\%) had diabetes, 79 (53\%) had hypertension and $31(21 \%)$ had both diseases. The average cluster size was 21 (SD 10). One hundred and twenty-seven patients $(85 \%)$ contributed data through the completion of the study. Twenty-two patients withdrew from the study; in 11, this was due to physician discontinuation of therapy. Of a total 1204 possible data collection time points corresponding to active study participation (ie, excluding data collection that would have occurred following a withdrawal), we collected data at 1154 time points (96\%). Of these, individuals at 397 time points were unexposed to the intervention and 757 were exposed.

Baseline demographic and disease data are presented in table 1. Sixty-four per cent of patients $(n=96)$ were women with a median age of 58 years (IQR 50-71). The median $\mathrm{HbAlc}$ in patients with diabetes was 9.3 (IQR $7.2-11.7)$ with $53 \%(\mathrm{n}=37)$ having a HbAlc $\geq 9 \%$. Twenty-two per cent of patients $(n=15 / 69)$ with diabetes had disease control at baseline. The median SBP in patients with hypertension was 135 (IQR 126-151). Fifty-nine per cent of patients $(n=61)$ with hypertension had disease control at baseline. For patients with two time points unexposed to the intervention, the ICC was 0.77 for SBP and 0.78 for DBP $(\mathrm{n}=86)$ among patients with hypertension and 0.97 for HbAlc among patients with diabetes $(\mathrm{n}=55)$.

\section{Continuous outcomes of $\mathrm{HbA1c}$ and blood pressure}

In adjusted analysis, among 73 patients with diabetes, there was a decrease in HbAlc of $0.35 \%$ with exposure to the intervention (figure $3 \mathrm{~A}$ ); however, CIs were wide ( $95 \%$ CI $-0.90 \%$ to $0.20 \%, \mathrm{p}=0.21$ ). Among 117 patients with hypertension, there was a $4.7 \mathrm{~mm} \mathrm{Hg}$ decrease in SBP with exposure to the intervention (95\% CI -8.9 to $-0.6 \mathrm{~mm} \mathrm{Hg}, \mathrm{p}=0.03$, figure $3 \mathrm{~B}$ ) and a $2.2 \mathrm{~mm} \mathrm{Hg}$ decrease in DBP (95\% CI -4.5 to $0.1 \mathrm{~mm} \mathrm{Hg}, \mathrm{p}=0.056)$.

In patients with diabetes with $\mathrm{HbA} 1 \mathrm{c} \geq 9 \%$ at baseline $(\mathrm{n}=37)$, relative to no intervention, exposure to the CHW intervention resulted in a decrease in $\mathrm{HbAlc}$ of $0.96 \%$ $(95 \% \mathrm{CI}-1.69 \%$ to $-0.23 \%, \mathrm{p}=0.01$, figure $3 \mathrm{~A})$. There was no evidence of a clinically significant intervention effect among patients with $\mathrm{HbAlc}<9 \%$ at baseline (estimate $0.11 \%, 95 \%$ CI $-0.62 \%$ to $0.84 \%, \mathrm{p}=0.76, \mathrm{n}=32$; $\mathrm{p}$ value for interaction: 0.04).

In patients with uncontrolled hypertension at baseline $(n=48)$, exposure to the intervention resulted in a 


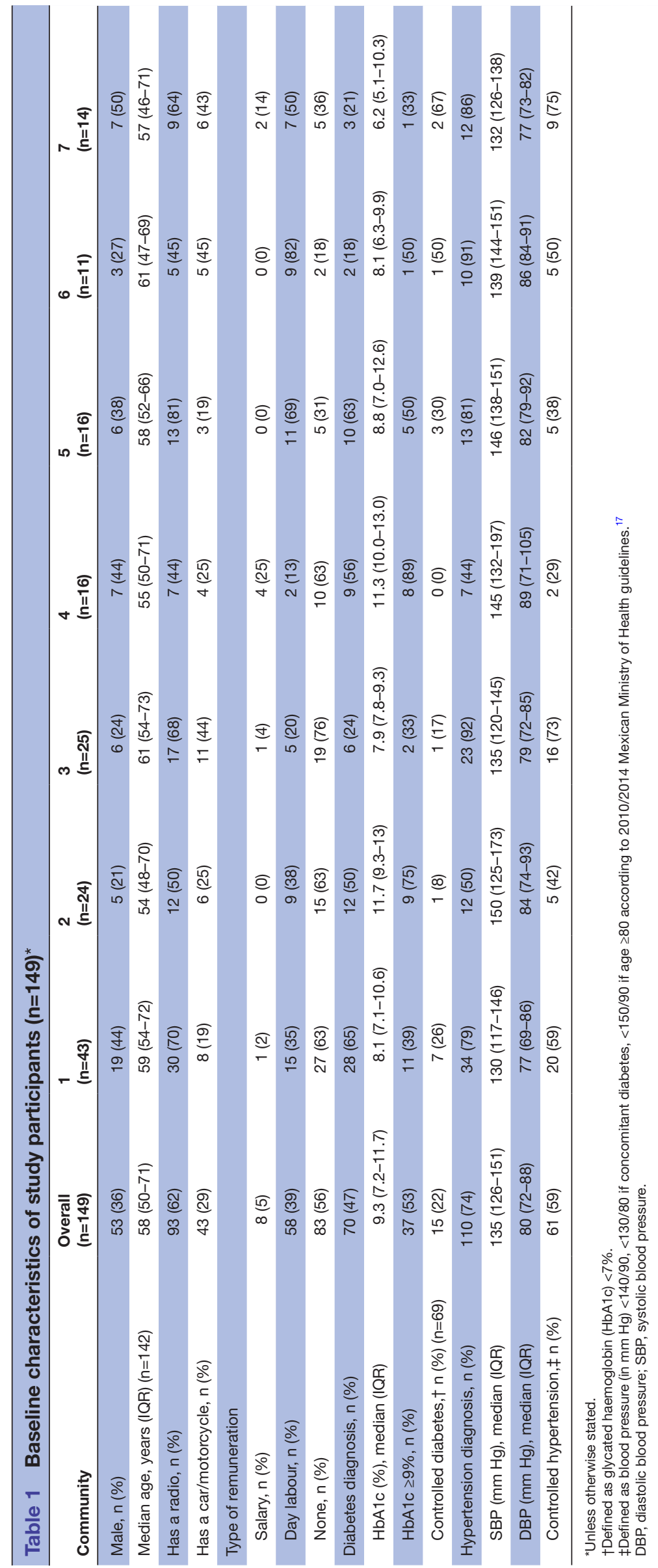



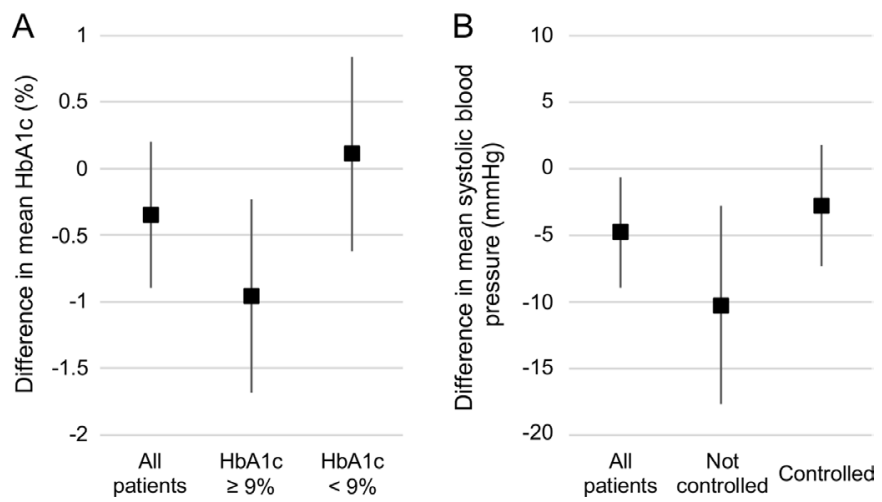

Figure 3 Diabetes and hypertension continuous outcomes. Adjusted mean difference between exposed and unexposed for glycated haemoglobin $(\mathrm{HbA1c})$ among patients with diabetes $(A)$ and average systolic blood pressure in patients with hypertension (B). Individual-level mixed effects analysis adjusting for time and cohort with clustering by individual and community presented as estimate (square) and 95\% Cls (lines). A. Diabetes outcomes among all patients ( $\mathrm{n}=73$ (543 time points)) and dichotomised between poorly controlled (HbA1c $\geq 9 \%, n=37$ (278)) and not poorly controlled $(\mathrm{HbA} 1 \mathrm{c}<9 \%, \mathrm{n}=32(247))$ at baseline. B. Hypertension outcomes among all patients ( $\mathrm{n}=117$ (869 time points)) and dichotomised between not controlled $(n=49(364))$ and controlled (blood pressure (in $\mathrm{mm} \mathrm{Hg}$ ) <140/90, <130/80 if concomitant diabetes, $<150 / 90$ if age $\geq 80$ according to 2010/2014 Mexican Ministry of Health guidelines, ${ }^{17} n=62$ (486)) at baseline. Four patients with diabetes and seven patients with hypertension not included in stratified analysis due to missing baseline control data.

$10.2 \mathrm{~mm} \mathrm{Hg}$ decrease in SBP (95\% CI -17.7 to $-2.8 \mathrm{~mm}$ $\mathrm{Hg}, \mathrm{p}=0.007$, figure $3 \mathrm{~B}$ ) relative to no intervention. Among patients with baseline hypertension control $(\mathrm{n}=62)$, the intervention was associated with a reduction of $2.8 \mathrm{~mm} \mathrm{Hg}(95 \% \mathrm{CI}-7.3$ to $1.8 \mathrm{~mm} \mathrm{Hg}, \mathrm{p}=0.23$; $\mathrm{p}$ value for interaction: 0.09). Similar results were observed for DBP: patients with uncontrolled baseline hypertension had a $4.6 \mathrm{~mm} \mathrm{Hg}$ decrease $(95 \%$ CI -8.5 to $-0.7 \mathrm{~mm} \mathrm{Hg}$, $\mathrm{p}=0.02$ ) and patients with baseline control $1.2 \mathrm{~mm} \mathrm{Hg}$
(95\% CI -3.9 to $1.5 \mathrm{~mm} \mathrm{Hg}, \mathrm{p}=0.39$; $\mathrm{p}$ value for interaction: 0.16$)$.

\section{Binary outcomes of controlled diabetes and hypertension}

In patients with diabetes, we observed a 2.7-fold increase in odds of disease control with receipt of the intervention, relative to none; however, CIs were wide and included one (adjusted OR 2.69, 95\% CI 0.72 to $10.14, \mathrm{p}=0.14, \mathrm{n}=73$, table 2). Among patients with hypertension, the intervention was associated with a 3.2-fold increase in the odds of disease control (adjusted OR 3.18, 95\% CI 1.55 to 6.55 , $\mathrm{p}=0.002, \mathrm{n}=117$ ).

Overall, we observed a greater effect of the intervention among patients with uncontrolled disease at baseline; however, small numbers limited power for statistical comparisons. The effect was larger among patients with uncontrolled diabetes at baseline (adjusted OR 5.35, $95 \%$ CI 0.89 to $32.17, \mathrm{p}=0.07, \mathrm{n}=54$ ) as compared with baseline disease control (adjusted OR 1.46, 95\% CI 0.06 to $37.26, \mathrm{p}=0.82, \mathrm{n}=15 ; \mathrm{p}$ value for interaction: 0.49 ). We also observed a greater effect among patients with uncontrolled hypertension at baseline (adjusted OR $6.28,95 \%$ CI 1.79 to $22.06, \mathrm{p}=0.004, \mathrm{n}=48$ ) than among patients with baseline controlled hypertension (adjusted OR 2.65, 95\% CI 0.99 to 7.12, $\mathrm{p}=0.053, \mathrm{n}=62$; $\mathrm{p}$ value for interaction: 0.29 ).

\section{Sensitivity analysis}

Results from sensitivity analyses (see online supplementary tables 1-5) were consistent with primary analyses and did not change interpretation of the findings.

\section{Adverse effects}

There were no adverse effects attributable to the CHW intervention.

\section{DISCUSSION}

Our findings suggest that when a CHW-led intervention, built around the values of accompaniment, ${ }^{32}$ is added to comprehensive primary care in rural Mexico, patients

Table 2 Intervention effectiveness, stratified by disease and baseline disease control

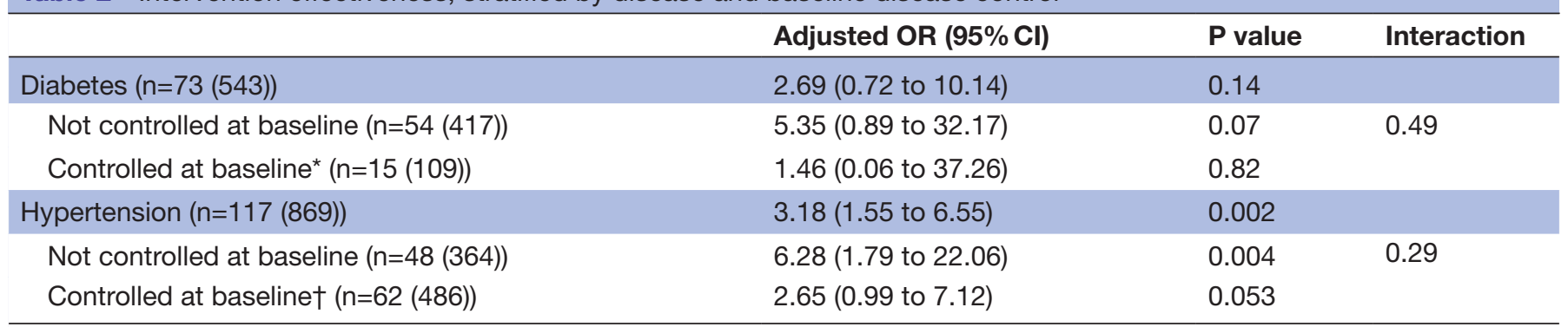

Individual-level mixed effects analysis adjusting for time and cohort with random intercepts to account for clustering by individual and community ( $\mathrm{n}=$ number of individual patients (number of time points)). Four patients with diabetes and seven patients with hypertension not included in stratified analysis due to missing baseline control data.

${ }^{*}$ Defined as glycated haemoglobin $(\mathrm{HbA} 1 \mathrm{c})<7 \%$.

†Defined as blood pressure (in $\mathrm{mm} \mathrm{Hg}$ ) <140/90, <130/80 if concomitant diabetes, $<150 / 90$ if age $\geq 80$ according to 2010/2014 Mexican Ministry of Health guidelines. ${ }^{17}$ 
with diabetes and hypertension can experience clinically significant improvements in markers of disease control. This includes a reduction in SBP among patients with hypertension, especially those with uncontrolled hypertension, and uncontrolled diabetes (HbAlc $\geq 9 \%)$. For patients with diabetes, precision was limited, and CIs were wider than for SBP. These findings suggest that the intervention was most effective for those in greatest need.

Our results are consistent with the growing literature of LMICs supporting the role of CHWs in improving cardiovascular disease risk. ${ }^{10}{ }^{12-15}$ Our study differs from many existing studies conducted among patients with newly diagnosed or uncontrolled disease in that we implemented the intervention in a population that included both controlled and uncontrolled patients already on pharmacologic treatment. The $10 \mathrm{~mm} \mathrm{Hg}$ reduction in SBP we observed among patients with uncontrolled hypertension is on par with recent cluster-randomised trials from India $(-8.9 \mathrm{~mm} \mathrm{Hg}),{ }^{10}$ Argentina $(-6.6 \mathrm{~mm}$ $\mathrm{Hg}),{ }^{13}$ Colombia and Malaysia $(-11.5 \mathrm{~mm} \mathrm{Hg}) .{ }^{15}$ Our study further adds to the literature by supporting the role for CHWs in patients with diabetes, where studies have produced promising but sometimes inconclusive results. ${ }^{8-10}$

The CHW intervention was implemented on top of a highly functional primary healthcare system. CHW presence likely contributed to more continuous medication adjustments, improved adherence and potentially lifestyle changes. That this intervention was most effective in those with poor control at baseline suggests that these tasks help such patients better use the care available to them, which is relevant to both high-income contexts and LMICs. In contrast, management of patients with baseline control requires maintenance, potentially achieved by CHW support and response to lapses in disease control. Although our results suggest a stronger effect among uncontrolled patients, our experience suggests that CHWs may learn and benefit from interactions with patients who are able to achieve clinical control. Additionally, disease control in NCDs like diabetes and hypertension can be dynamic, fluctuating over time for an individual patient and posing an increased risk of complications. ${ }^{33}$ For these reasons, we advocate CHW interventions targeting all patients and, at minimum, the most socially vulnerable, not only those with the worst control. We anticipate that further addressing social determinants, such as the provision of medically tailored meals, ${ }^{34}$ stable housing, income assistance and mental health supports, will yield even greater results.

The clinical benefits of CHW interventions in patients with uncontrolled diabetes or hypertension are similar to those associated with the addition of medications to treatment regimens. ${ }^{65}$ The magnitude of intervention effect observed could significantly reduce diabetes/ hypertension-associated morbidity and mortality. By allocating healthcare financing to human-mediated inputs such as paying, training and supporting CHWs in addition to medication supplies, there are ancillary benefits for society including empowerment of women, economic stimulation and decreased unemployment. Any approach that aims to comprehensively address the social determinants of disease and improve outcomes will require new investments in order to build and finance truly functional health systems. The emergence of mobile health technologies may allow for further programmatic and health systems coordination, targeting attrition and providing real-time feedback, with further study needed to assess their role as well as ideal models of CHW task load and supervision structure.

We evaluated this intervention in the context of a programmatic stepped roll-out, in which patients initiated the intervention during the follow-up period. This approach allowed us to rule out confounding by stable individual-level characteristics and adjust for underlying time trends. Secular trends and regression to the mean are common concerns in longitudinal analyses; however, adjustment for time would minimise these potential biases. The high correlation of adjacent measurements, together with sensitivity analyses that supported primary findings and effect estimates in the same direction in both strata, suggest that regression to the mean is unlikely to explain the results of stratified analyses. However, the small sample size may have limited statistical comparisons across subgroups. The use of objective markers of disease control, HbA1c and blood pressure, support the robustness of our results-any influence being observed (ie, Hawthorne effect) would likely be apparent in both arms, and therefore would not explain our study findings. Our CHW intervention set minimum standards for visit frequency, but was not designed to assess visit length, frequency or number of tasks per visit and did not map individual CHWs to patients. These programme characteristics may impact the success of a CHW intervention and future studies could facilitate optimisation. A lack of data that linked CHWs to patients also precluded adjustment for clustering at the CHW level, which would be important if some CHWs were more or less effective. However, standardisation of training, practices and supervision should have limited variability among CHWs. Our intervention was evaluated in a single rural, remote setting, with limited phone and internet access, dirt roads and long distances to higher levels of care. The success of the intervention despite these barriers and with comparable evidence from LMICs suggest that the findings may be generalisable to other remote, rural settings.

In conclusion, we demonstrate that an integrated CHW-led intervention targeting NCDs in rural Mexico can improve measures of disease in patients with uncontrolled diabetes and hypertension. Programmes and health systems aiming to improve care of patients with NCDs may consider this study as supportive evidence for the addition of professionalized CHWs to strengthen rural primary care systems in LMICs. 
Author affiliations

${ }^{1}$ Division of Global Health Equity, Brigham and Women's Hospital, Boston,

Massachusetts, USA

${ }^{2}$ Compañeros en Salud, Ángel Albino Corzo, Mexico

${ }^{3}$ Department of Global Health and Social Medicine, Harvard Medical School, Boston, Massachusetts, USA

${ }^{4}$ Partners In Health, Boston, Massachusetts, USA

${ }^{5}$ Division of Pediatric Hospital Medicine, Department of Pediatrics, University of California San Francisco School of Medicine, San Francisco, California, USA

Acknowledgements The authors thank the patients for their participation in the study and the CHWs for their dedicated service to their patients. We thank Aracel Gomez Velasco, Edgar Gomez Velasco, Ramiro Cortez Castro, Salomon Benites Santiago and Nick Seymour for their assistance in data collection. We thank Jafet Arrieta and Hector Carrasco for their contributions to the design and implementation of the original CHW program. The authors additionally thank the Global Health Research Core at the Department of Global Health and Social Medicine at Harvard Medical School for their support.

Contributors DTW oversaw data collection, carried out the analysis, produced figures and tables, led the literature search and drafted the manuscript. MFF, HF, LP, PMN and DP contributed to study design. JK and KR led data collection teams. $\mathrm{DTW}, \mathrm{RB}, \mathrm{ZG}, \mathrm{JM}$ and PMN oversaw the data collection process and ensured data quality. MFF was the lead methodologist, designed the analysis and critically reviewed the manuscript. JK contributed to the literature search and manuscript preparation. PMN and DP were responsible for the original conceptualisation, study oversight and critical review of the manuscript as principal investigators. All authors contributed to interpretation of the work, editing of the manuscript, final approval of this version and agree to be accountable for all aspects of the work.

Funding This study was partially funded by a grant from the Harvard Global Health Institute. DTW's travel costs were supported by the Doris and Howard Hiatt Residency in Global Health Equity and Internal Medicine at the Brigham and Women's Hospital. JK's travel and living costs were partially supported by a Wesleyan Summer Grant from Wesleyan University.

Disclaimer The funders did not have any role in study design; in the collection, analysis and interpretation of the data; in the writing of the report or in the decision to submit the paper for publication.

Competing interests None declared.

Patient consent for publication Not required.

Ethics approval This study was reviewed and approved by institutional review boards of the Brigham and Women's Hospital (Partners Human Research Committee, Boston, USA) and the Instituto Tecnológico de Monterrey (Monterrey, México).

Provenance and peer review Not commissioned; externally peer reviewed.

Data availability statement De-identified primary data and a technical appendix are available on reasonable request from the authors.

Open access This is an open access article distributed in accordance with the Creative Commons Attribution Non Commercial (CC BY-NC 4.0) license, which permits others to distribute, remix, adapt, build upon this work non-commercially, and license their derivative works on different terms, provided the original work is properly cited, appropriate credit is given, any changes made indicated, and the use is non-commercial. See: http://creativecommons.org/licenses/by-nc/4.0/.

\section{ORCID iDs}

Devin T Worster http://orcid.org/0000-0002-6211-3794

Molly F Franke http://orcid.org/0000-0002-4890-5728

\section{REFERENCES}

1 World Health Organization. Noncommunicable diseases, 2018. Available: https://www.who.int/en/news-room/fact-sheets/detail/ noncommunicable-diseases [Accessed 1 Jan 2020].

2 Di Cesare M, Khang Y-H, Asaria P, et al. Inequalities in noncommunicable diseases and effective responses. Lancet 2013;381:585-97.

3 Mishra SR, Neupane D, Preen D, et al. Mitigation of noncommunicable diseases in developing countries with community health workers. Global Health 2015;11:43.
4 Khetan AK, Purushothaman R, Chami T, et al. The effectiveness of community health workers for CVD prevention in LMIC. Glob Heart 2017;12:233-43.

5 Stratton IM, Adler Al, Neil HA, et al. Association of glycaemia with macrovascular and microvascular complications of type 2 diabetes (UKPDS 35): prospective observational study. BMJ 2000;321:405-12.

6 Law MR, Morris JK, Wald NJ. Use of blood pressure lowering drugs in the prevention of cardiovascular disease: meta-analysis of 147 randomised trials in the context of expectations from prospective epidemiological studies. BMJ 2009;338:b1665

7 Alaofè $\mathrm{H}$, Asaolu I, Ehiri J, et al. Community health workers in diabetes prevention and management in developing countries. Ann Glob Health 2017;83:661-75.

8 Morris-Paxton AA, Rheeder P, Ewing R-MG, et al. Detection, referral and control of diabetes and hypertension in the rural eastern Cape Province of South Africa by community health outreach workers in the rural primary healthcare project: health in every hut. Afr J Prim Health Care Fam Med 2018;10:a1610.

9 Farzadfar F, Murray CJL, Gakidou E, et al. Effectiveness of diabetes and hypertension management by rural primary health-care workers (Behvarz workers) in Iran: a nationally representative observational study. Lancet 2012;379:47-54.

10 Khetan A, Zullo M, Rani A, et al. Effect of a community health workerbased approach to integrated cardiovascular risk factor control in India. Glob Heart 2019;14:355-65.

11 Anand TN, Joseph LM, Geetha AV, et al. Task sharing with nonphysician health-care workers for management of blood pressure in low-income and middle-income countries: a systematic review and meta-analysis. Lancet Glob Health 2019;7:e761-71.

12 Jafar TH, Hatcher J, Poulter N, et al. Community-based interventions to promote blood pressure control in a developing country: a cluster randomized trial. Ann Intern Med 2009;151:593-601.

$13 \mathrm{He}$ J, Irazola V, Mills KT, et al. Effect of a community health workerled multicomponent intervention on blood pressure control in low-income patients in Argentina: a randomized clinical trial. JAMA 2017;318:1016-25.

14 Neupane D, McLachlan CS, Mishra SR, et al. Effectiveness of a lifestyle intervention led by female community health volunteers versus usual care in blood pressure reduction (COBIN): an openlabel, cluster-randomised trial. Lancet Glob Health 2018;6:e66-73.

15 Schwalm J-D, McCready T, Lopez-Jaramillo P, et al. A communitybased comprehensive intervention to reduce cardiovascular risk in hypertension (hope 4). Lancet 2019;394:1231-42.

16 Institute for Health Metrics and Evaluation (IHME). Mexico profile, 2018. Available: http://www.healthdata.org/mexico [Accessed 1 Jan 2020].

17 Secretaría de Salud, México. Programa de acción específico: prevención y control de la obesidad y riesgo cardiovascular 2013-2018, 2014. Available: http://www.cenaprece.salud.gob.mx/ descargas/pdf/PAE_PrevencionControlObesidadRiesgoCardiovas cular2013_2018.pdf [Accessed 11 Mar 2019].

18 Instituto Nacional de Salud Pública y Secretaría de Salud, México. Encuesta nacional de salud y nutrición de medio camino 2016 (ENSANUT 2016), 2016. Available: http://transparencia.insp.mx/ 2017/auditorias-insp/12701_Resultados_Encuesta_ENSANUT_ MC2016.pdf [Accessed 11 Mar 2019].

19 Secretaría de Salud M. Estrategia nacional para la prevención y el control del sobrepeso la obesidad y la diabetes, 2013. Available: https://www.gob.mx/cms/uploads/attachment/file/200355/ Estrategia_nacional_para_prevencion_y_control_de_sobrepeso_ obesidad y diabetes.pdf [Accessed 11 Mar 2019].

20 Balcazar H, Perez-Lizaur AB, Izeta EE, Escalante Izeta E, et al. Community health Workers-Promotores de Salud in Mexico: history and potential for building effective community actions. J Ambul Care Manage 2016;39:12-22.

21 Consejo Nacional de Evaluación de la Política de Desarrollo Social (CONEVAL). Medición de la pobreza en México y en las Entidades Federativas, 2016. Available: https://coneval.org.mx/Medicion/MP/ Paginas/Pobreza_2016.aspx [Accessed 6 Jul 2019].

22 Newman PM, Franke MF, Arrieta J, et al. Community health workers improve disease control and medication adherence among patients with diabetes and/or hypertension in Chiapas, Mexico: an observational stepped-wedge study. BMJ Glob Health 2018;3:e000566.

23 Behforouz HL, Farmer PE, Mukherjee JS. From directly observed therapy to accompagnateurs: enhancing AIDS treatment outcomes in Haiti and in Boston. Clin Infect Dis 2004;38:S429-36.

24 Franke MF, Kaigamba F, Socci AR, et al. Improved retention associated with community-based accompaniment for antiretroviral therapy delivery in rural Rwanda. Clin Infect Dis 2013;56:1319-26. 
25 National Quality Forum. Comprehensive diabetes care: Hemoglobin A1c (HbA1c) poor control (>9.0\%), 2017. Available: http://www. qualityforum.org/QPS/QpsMeasureExport.aspx?exportType=pdf\& exportFrom=s\&measurelDs=1225 [Accessed 11 Mar 2019].

26 Teerenstra S, Taljaard M, Haenen A, et al. Sample size calculation for stepped-wedge cluster-randomized trials with more than two levels of clustering. Clin Trials 2019;16:225-36.

27 Hussey MA, Hughes JP. Design and analysis of stepped wedge cluster randomized trials. Contemp Clin Trials 2007;28:182-91.

28 Hemming K, Haines TP, Chilton PJ, et al. The stepped wedge cluster randomised trial: rationale, design, analysis, and reporting. BMJ 2015;350:h391.

29 Hemming K, Taljaard M, Forbes A. Analysis of cluster randomised stepped wedge trials with repeated cross-sectional samples. Trials 2017;18:101.

30 Capanu M, Gönen M, Begg CB. An assessment of estimation methods for generalized linear mixed models with binary outcomes. Stat Med 2013;32:4550-66.
31 Secretaría de Salud, Mexico. PROYECTO de Norma Oficial Mexicana PROY-NOM-030-SSA2-2017, Para La prevención, detección, diagnóstico, tratamiento $\mathrm{Y}$ control de la hipertensión arterial sistémica, 2017. Available: http://www.dof.gob.mx/nota detalle. php?codigo $=5480159 \&$ fecha $=19 / 04 / 2017$ [Accessed 11 Mar 2019].

32 Palazuelos D, Farmer PE, Mukherjee J. Community health and equity of outcomes: the partners in health experience. Lancet Glob Health 2018;6:e491-3.

33 Rothwell PM, Howard SC, Dolan E, et al. Prognostic significance of visit-to-visit variability, maximum systolic blood pressure, and episodic hypertension. Lancet 2010;375:895-905.

34 Mozaffarian D, Mande J, Micha R. Food is Medicine-The promise and challenges of integrating food and nutrition into health care. JAMA Intern Med 2019:179:793-5.

35 George CM, Brujin LL, Will K, et al. Management of blood glucose with Noninsulin therapies in type 2 diabetes. Am Fam Physician 2015;92:27-34 